\title{
Right atrial volume indexed by cardiovascular magnetic resonance as a predictor of mortality in patients with heart failure with reduced ejection fraction
}

\author{
Alexander Ivanov ${ }^{1 *}$, Ambreen Mohamed ${ }^{1}$, Ahmed Asfour ${ }^{1,3}$, Marc N Katz ${ }^{1}$, Christine Li ${ }^{1}$, Jean Y Ho ${ }^{1}$, \\ Michelle Gorbonosov ${ }^{1}$, On Chen ${ }^{2}$, Joshua Socolow ${ }^{1}$, Sorin Brener ${ }^{1}$, John Heitner ${ }^{1}$
}

From 19th Annual SCMR Scientific Sessions

Los Angeles, CA, USA. 27-30 January 2016

\section{Background}

Right Atrial Volume Indexed (RAVI) measured by echocardiogram was identified to be an independent predictor of morbidity in patients with heart failure (HF) with reduced ejection fraction (HFrEF). Meta-Analysis Global Group in Chronic heart failure (MAGGIC) risk score is a robust tool in predicting mortality in HF patients. The aim of this study is to evaluate RAVI by cardiac magnetic resonance (CMR) imaging as an independent predictor of all-cause mortality in patients with HFrEF and to compare it with the validated risk score

\section{Methods}

We identify 488 patients with left ventricular ejection fraction (LVEF) $<35 \%$ assessed by CMR. We excluded patients undergoing open-heart surgery, severe valvular disease and patients with inadequate imaging. Right atrial volume was calculated based on a measured area in two and four chamber views utilizing a validated equation and indexed to body surface area. MAGGIC risk score was calculated using an online calculator. Follow-up information was acquired via phone call questionnaire and social security death index in patients not able to be reached directly. Our primary outcome was all-cause mortality or an appropriate implantable cardioverter defibrillator therapy.

\section{Results}

Two hundred forty four patients (mean age $60 \pm 15$; 33\% women) were followed for a mean period of 2.1 years.

${ }^{1}$ Cardiology, New York Methodist Hospital, Brooklyn, NY, USA

Full list of author information is available at the end of the article
Thirty-two patients (13\%) had a primary outcome. The mean RAVI was $53 \pm 26 \mathrm{ml} / \mathrm{m} 2$. The RAVI was significantly larger in patients with an event than without $(75.9 \pm 31 \mathrm{ml} / \mathrm{m} 2$ vs. $49 \pm 23 \mathrm{ml} / \mathrm{m} 2, \mathrm{p}<0.001)$. The mean MAGGIC score was $19.7 \pm 7$ (mean one-year mortality $10.2 \%$ ). RAVI assessed continuously was an independent predictor of mortality controlled for MAGGIC risk, right ventricular ejection fraction (RVEF[JH1]) with $\mathrm{HR}$ 1.02(1.01-1.03), $\mathrm{p}=0.002$. RAVI $(0.76 \pm 0.08)$ has a greater $\mathrm{C}$ statistic than LVEF $(0.55 \pm 0.1, \mathrm{p}<0.004)$, left atrial volume indexed $(0.64 \pm 0.1, \mathrm{p}<0.025)$ and has a trend towards greater $\mathrm{C}$ statistics than RVEF $(0.65 \pm 0.11, \mathrm{p}<$ 0.06). The addition of RAVI to the MAGGIC risk score significantly reclassify risk (integrated discrimination improvement by $9 \%, \mathrm{p}<0.0015$ and category-free net reclassification improvement by $58 \%, \mathrm{p}<0.0029$ ).

\section{Conclusions}

RAVI measured by CMR is an independent predictor of mortality in patients with HFrEF. The addition of RAVI to MAGGIC score significantly reclassify risk.

\section{Authors' details}

'Cardiology, New York Methodist Hospital, Brooklyn, NY, USA. ${ }^{2}$ Cardiology, Maymonides Medical Center, Brooklyn, NY, USA. ${ }^{3}$ Department of Cardiovascular Medicine, Cairo University, Cairo, Egypt.

Published: 27 January 2016

doi:10.1186/1532-429X-18-S1-033

Cite this article as: Ivanov et al: Right atrial volume indexed by cardiovascular magnetic resonance as a predictor of mortality in patients with heart failure with reduced ejection fraction. Journal of Cardiovascular Magnetic Resonance 2016 18(Suppl 1):O33. 